\title{
Research Paper: The Neuro-Occupation Model for Occupational Therapy: A Correlation Study
}

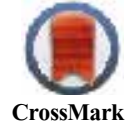

\author{
Seyed Alireza Derakhshanrad ${ }^{1,2 *}$, Emily Piven $^{3}$
}

1. Department of Occupational Therapy, School of Rehabilitation Sciences, Shiraz University of Medical Sciences, Shiraz, Iran

2. Rehabilitation Sciences Research Center, Shiraz University of Medical Sciences, Shiraz, Iran.

3. Health Matters First of Florida Incorporation, Oakland, United States.

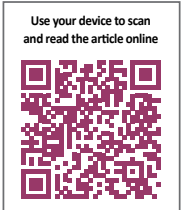

Citation: Derakhshanrad SA, Piven E. The Neuro-Occupation Model for Occupational Therapy: A Correlation Study. Iranian Rehabilitation Journal. 2017; 15(4):351-358. https://doi.org/10.29252/NRIP.IRJ.15.4.351

: https://doi.org/10.29252/NRIP.IRJ.15.4.351

\section{Article info:}

Received: 07 Jun. 2017

Accepted: 19 Oct. 2017

\section{Keywords:}

Cerebrovascular strokes, Cognition, Neuroscience, Occupations, Theoretical models

\begin{abstract}
Objectives: The Neuro-occupation model has been referenced as the single occupational therapy conceptual framework that considers the interaction of three underpinning variables of Intention, Meaning, and Perception that explains how occupational performance is shaped. To date, studies have focused on the qualitative relationships between the variables. Quantitative studies that focus on the relationships among variables are lacking, begging the question as to whether the model is well-conceptualized. Extending prior work on the Neuro-occupation model, the aim of this quantitative study is to test the model by investigating correlations among the key variables of the model.
\end{abstract}

Methods: This is a correlational study by a convenience sample of 25 cognitively-oriented patients with strokes recruited from three rehabilitation facilities in Shiraz, Iran. The participants were evaluated using three standardized instruments to measure the variables: 1 . Adapted Achievement Motivation Questionnaire, 2. Connor-Davidson Resilience Scale, and 3. Canadian Occupational Performance Measure. To control the effects of months post-stroke and cognitive functioning, the partial correlation test was used to explore relationships among the variables.

Results: The correlational analysis indicated significant positive relationships among the variables of intention, meaning, and perception. The partial correlations showed acceptable correlation coefficients $(r \geq 0.45, \mathrm{P}<0.05)$.

Discussion: The Neuro-occupation model is a well-conceptualized framework, which can assist occupational therapists in understanding the design of occupational performance.

\section{Introduction}

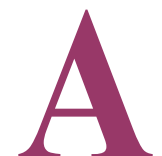

practitioner should develop skills at merging theory with practice to become a sophisticated occupational therapist.
Practice 'refers to what occupational therapists do in their professional roles' [1], and theory 'can be used to explain phenomena as well as predict the likely outcomes of changes to those phenomena' [1]. The exact nature of outcome changes cannot be predicted; how-

\section{* Corresponding Author:}

Seyed Alireza Derakhshanrad, PhD

Address: Department of Occupational Therapy, School of Rehabilitation Sciences, Shiraz University of Medical Sciences, Shiraz, Iran.

Tel: +98 (917) 7181002

E-mail:derakhshan@sums.ac.ir 
ever, they are always expected to happen [1]. Considered as theoretical-driven frameworks, models can help in clarifying phenomena by specifying the related concepts or variables such that specific outcomes can be expected to emerge [2].

It is essential that models should be treated seriously so that the practitioner can make well-reasoned and practical decisions on the use of models to inform practice [1]. The Neuro-occupation model is of particular interest to this study [3]. According to the conceptual framework, assumption cognition is a mental process in which three concepts or variables are known as Intention, Meaning, and Perception (IMP), are considered related in such a way as to delineate behavioral outcomes expected to emerge. Under the influences of variables mentioned above, individuals understand their world by intending to act, making meaning through their action, and raising perceptual awareness [4].

Producing evidence for clarifying the relationships among the variables of intention, meaning, and perception is so crucial that cognition was alleged to be further explored through that clarification [3]. Accordingly, various studies have focused on this speculation as to whether the relationships among the IMP variables could describe the emerging human performance. These relationships were declared crucial in establishing appropriate behavior in a middle-aged male patient with alcoholism when he: a. Made an intentional decision to control intemperance, $b$. Found meaning in abstinence, and $c$. Perceived himself capable of choosing a new lifestyle without substance abuse [5]. Another study pronounced the relationships critical in developing acceptable behavior of a 78-year-old hospitalized patient with stroke who demonstrated aggressive, non-adherent behavior [6].

The refined relationships among the client's IMP enabled him to progress to a. Decide intentionally to selfextinguish aggressive interactions, $b$. Find meaning in reinstating social behaviors, and c. Perceive himself capable of expanding suitable occupational patterns [6]. Additionally, three qualitative research studies gave credence to the relationships among the IMP variables. One study compared two matched male patients with strokes, both were identified as highly resilient, whereby the findings provided evidence for the proposed relationships among the IMP variables [7]. Subsequently, a study involved comparison of a female with stroke, who demonstrated low resilience behavior, to another female with stroke who demonstrated high resilience behavior. The findings showed that the relationships among the IMP variables have been either effective or ineffective, of which the results would lead to two different types of behavior called flexible or inflexible [8]. There was a study of 11 patients with strokes who were all highly resilient and seemed to be invested effectively in harmonious relationships among deliberate decisions, admissible meanings, and realistic perceptions [9].

Few studies performed on this model provided proof that the Neuro-occupation model has a well-conceptualized framework to be adopted by practitioners in their daily practice. Therefore, it could be argued that the model is still on its way to be recognized widely. Nevertheless, no quantitative research has been found confirming the technically feasible relationships among three variables of intention, meaning, and perception.

Based on these aforementioned points, the purpose of this correlational study was to provide evidence for the Neuro-occupation model, by finding the answer to the question of whether the IMP variables have statistically significant correlational relationships with each other. Specifically, we tested the hypothesis that there would be statistically significant correlations among IMP variables of a sample of patients with strokes, thus supporting the claim that the IMP variables were well-chosen concepts for the model to be conceptualized.

\section{Methods}

\section{Study design}

This was a non-experimental, correlational study to identify the relationships among variables, without manipulation by researchers. This study will provide an advantageous perspective for future experimental studies [10].

\section{Participants}

The statistical population included patients with cerebrovascular strokes. The following inclusion criteria guided a convenience sampling method: a. Cognitivelyoriented patients who scored $\geq 21$ on Mini-Mental State Examination, b. Those who had a post-stroke time between 3 to 24 months, and c. Patients aged from 25 to 70 years. These ranges were chosen because the researchers assumed that it might have consisted of a homogeneous sample from the targeted phenomenon point of view that was under investigation. The project used a convenience sample that was recruited from three main rehabilitation facilities in the city of Shiraz, Iran.

A study on developing a motivation questionnaire for a sample of stroke survivors provided sample size guide- 
lines, to calculate the minimum required a sample size of 25 patients, using the sample size equation, where $\alpha=0.05, \beta=0.2, d=4.5$, and mean \pm SD of the motivation questionnaire for the study population were $74.3 \pm 8.1$ [11]. In the interest of obtaining useful data, three standardized questionnaires were used to measure three abstract concepts including IMP variables.

$$
N=\frac{\left(Z_{1-\alpha / 2}+Z_{1-\beta}\right)^{2} S^{2}}{d^{2}}
$$

\section{Research instruments}

\section{Adapted Achievement Motivation Questionnaire (AAMQ)}

The concept of intention was measured by a questionnaire called Adapted Achievement Motivation Questionnaire (AAMQ) [11]. The questionnaire contained a total of 28 incomplete statements along with four suggested items used by examinee to complete the statements. The total score should be within the range of 28 to 112 ; the higher scores imply the higher motivation for recovery. Cronbach's alpha of 0.946 proved the questionnaire to be highly reliable for use in Iranian population [11].

\section{Connor-Davidson Resilience Scale (CD-RISC)}

Being associated with meaning, resilience is a personal ability and strength that has been acknowledged to help individuals put value on their experiences by finding meaning in their troubles [12]. Specifically, resilience was also reported to be a useful concept to explore how meaningful a life could be as experienced after stroke [13]. For these reasons, the concept of meaning was measured by a questionnaire called Connor-Davidson Resilience Scale (CD-RISC) [14]. The questionnaire was comprised of 25 items based on a 5-point Likert scale, ranging from 0 (totally false) to 4 (always true). The total score would be within 0 to 100 , whereas higher scores implied higher resilience. Being validated for a sample of Iranian patients with strokes, the test has proven to be reliable with Cronbach's alpha of 0.892 [15].

\section{Canadian Occupational Performance Measure (COPM)}

The concept of perception was sought by a semistructured interview assessment known as Canadian Occupational Performance Measure (COPM), which was used to evaluate a client's perception of his or her occupational performance [16]. Administrating the COPM is a four-step process including: a. Interviewing the client to determine the problems in doing activities of self-care, productivity, and leisure, b. Asking the client to determine how important each recognized problem was to him or her using a 1 (least important) to 10 (most important) scale, c. Prioritizing the five most urgent problems on the basis of the importance rating from the previous step, and $\mathrm{d}$. Then asking the client to rate his or her ability to perform the five most urgent problems and rate the satisfaction with performance on a 1-10 scale. Consequently, there would be two scores- one for performance and one for satisfaction. Performance and satisfaction scores can be obtained from the below equations:

sum of the scores given to the best of ability

Performance $=\frac{\text { to perform the five most urgent problems }}{5}$

sum of the scores given to the satisfaction with Satisfaction $=\frac{\text { performing the five most urgent problems }}{5}$

The total score for each part would be within 1 to 10 ; higher scores implied that the client perceived to be more satisfied and capable of doing the urgent daily activities. COPM was translated into Persian and was determined to be suitable for use in Iranian population [17]. The testretest reliability of the Persian version of COPM showed acceptable results for performance $(\mathrm{r}=0.80, \mathrm{P}<0.05)$ and satisfaction $(\mathrm{r}=0.84, \mathrm{P}<0.05)$ sections [18].

\section{Data analysis}

The test results were analyzed using the Statistical Package for Social Sciences (SPSS), version 23. Descriptive statistics were calculated. The KolmogorovSmirnoff test shows that scores on all study variables resulting from AAMQ, CD-RISC, and COPM (performance and satisfaction) have a normal distribution; as a result, the relationships were explored using a parametric test. While controlling for the effects of months post-stroke and cognitive functioning, thought to have potential to influence the relationships, partial correlation test was used to explore relationships among study variables. Partial correlation is an extension of Pearson correlation that allows researchers to remove the influence of confounding variables statistically [19].

\section{Procedure}

Before commencing the study, ethical clearance was sought from the ethical committee of Shiraz University 
of Medical Sciences, Iran. All participants gave their consent to participate in the study. Sampling was conducted over a 5-month period from January to May 2017. Reaching the pre-determined sample size, the project completed with 25 participants who fulfilled the inclusion criteria. First, AAMQ and CD-RISC were given to the participants. They were also provided with enough time to respond to the inquiries. Then, COPM was administered by the researcher together with the client.

\section{Results}

Creating a convenience sample method, a total of 25 cognitively-oriented patients with strokes participated in this study. The mean age was $53.2(\mathrm{SD}=11.3$, range: 25-70). There was no statistically significant difference between males and females mean ages $(\mathrm{P}=0.062)$. The total scale means and other descriptive information for the research instruments were: $1 . \mathrm{AAMQ}=91.4(\mathrm{SD}=8.4$, range: $76-104) ; 2$. $\mathrm{CD}-\mathrm{RISC}=74.7(\mathrm{SD}=15.8$, range:

Table 1. Descriptive information for the study participants

\begin{tabular}{|c|c|c|c|c|c|}
\hline & N (\%) & Mean & SD & Min & Max \\
\hline \multicolumn{6}{|c|}{ Age (year) } \\
\hline Male & 14.56 & 57 & 9.2 & 37 & 70 \\
\hline Female & 11.44 & 48.5 & 12.4 & 25 & 70 \\
\hline \multicolumn{6}{|l|}{ Months post-stroke } \\
\hline Male & 14.56 & 9.1 & 4.2 & 3 & 16 \\
\hline Female & 11.44 & 9.6 & 6.2 & 3 & 24 \\
\hline MMSE & & 26.2 & 2.9 & 21 & 30 \\
\hline \multicolumn{6}{|c|}{ AAMQ } \\
\hline Male & 14.56 & 92.1 & 8.7 & 77 & 104 \\
\hline Female & 11.44 & 89.6 & 8.2 & 76 & 100 \\
\hline Left hemiplegic subjects & 15.60 & 91.5 & 9.7 & 76 & 104 \\
\hline Right hemiplegic subjects & 10.40 & 90.3 & 6.6 & 79 & 100 \\
\hline \multicolumn{6}{|c|}{ CD-RISC } \\
\hline Male & 14.56 & 78.2 & 13.1 & 56 & 98 \\
\hline Female & 11.44 & 70.2 & 18.3 & 42 & 98 \\
\hline Left hemiplegic subjects & 15.60 & 72.7 & 17.7 & 42 & 98 \\
\hline Right hemiplegic subjects & 10.40 & 78.4 & 12.5 & 61 & 98 \\
\hline \multicolumn{6}{|c|}{ COPM (performance) } \\
\hline Male & 14.56 & 4.1 & 1.7 & 1 & 6.6 \\
\hline Female & 11.44 & 4.3 & 2 & 1.4 & 7.2 \\
\hline Left hemiplegic subjects & 15.60 & 4.1 & 1.8 & 1.4 & 7.1 \\
\hline Right hemiplegic subjects & 10.40 & 4.3 & 1.9 & 1 & 7.2 \\
\hline \multicolumn{6}{|c|}{ COPM (satisfaction) } \\
\hline Male & 14.56 & 4.1 & 1.7 & \multicolumn{2}{|c|}{$\begin{array}{cc}1 & 7.4 \\
\text { Irantan Benabmiltationlournal }\end{array}$} \\
\hline Female & 11.44 & 3.7 & 2.3 & 1 & 8.3 \\
\hline Left hemiplegic subjects & 15.60 & 3.5 & 1.7 & 1 & 5.6 \\
\hline Right hemiplegic subjects & 10.40 & 4.9 & 2.3 & 1 & 8.3 \\
\hline
\end{tabular}

AAMQ: Adapted Achievement Motivation Questionnaire; CD-RISC: Connor-Davidson Resilience Scale; COPM: Canadian Occupational Performance Measure; MMSE: Mini-Mental State Examination; SD: Standard Deviation 
Table 2. Partial correlations among the study variables

\begin{tabular}{|c|c|c|c|c|}
\hline Control Variables (Months Post-Stroke \& Cognitive Function) & 1 & 2 & 3 & 4 \\
\hline AAMQ & - & & & \\
\hline CD-RISC & $0.676^{*}$ & - & & \\
\hline COPM (performance) & $0.585^{\dagger}$ & $0.450 \ddagger$ & - & \\
\hline COPM (satisfaction) & $0.617+$ & $0.580+$ & $0.845^{*}$ & - \\
\hline
\end{tabular}

42-98); 3. $\mathrm{COPM}$ (performance $)=4.2(\mathrm{SD}=1.8$, range: 1-7.2); and 4. COPM (satisfaction $)=3.9(\mathrm{SD}=1.9$, range: 1-8.3) (Table 1).

The correlational analysis of the results indicated significant positive relationships among the IMP variables. The partial correlations showed significant correlations $(\mathrm{r} \geq 0.45)$ among the study variables $(\mathrm{P}<0.05)$ while controlling for scores on months post-stroke and cognitive functioning (Table 2).

\section{Discussion}

The primary focus of this study was to test the Neurooccupation model through examining its central premise that IMP are three principal variables interacting with each other for change in cognition to occur. The results of that interactive cognition lead individuals to make decisions, find meaning, and alter perception concerning their performance of occupations and engagement in the environment. As explained in the introduction section of the paper, few attempts have been made to explore the relationships among the IMP variables, yet the previous studies have been qualitative. Therefore, it was considered that a quantitative research would be advantageous for examining the correlations among the IMP concepts, thus giving credence to the critical roles of IMP variables in shaping an interactive cognitive-oriented process.

The overall results of the study indicated that the IMP variables had significant correlations with each other. In line with this was Crabtree's notion that 'clients' intentions and assessments about their performance, and their perception of the outcomes of their performance are embedded and best expressed in the process of occupational performance [20]. Although not mentioning any specific theory or conceptual framework in his paper, Crabtree's non-empirical publication was found to be the sole work to explain the importance of IMP variables interactive feedback that established and changed occupational performance [20]. In reviewing the occupational therapy literature, except studies by Lazzarini and Crabtree [3, 20], no other case did these authors find the IMP concepts to be considered in combination with each other. However, considered separately, intention, meaning, or perception has been acknowledged to have major contributions to the construct of occupational performance. For example, one's perception is considered to be a major determinant of occupational performance [21].

Also, the concept of intention was presumed to be 'a critical element in human performance and, by extension, fundamental to occupational performance and occupational therapy.' [22]. Furthermore, meaning-making has been reported to have a central place in shaping occupational performance [23]. Except for the Neurooccupation model, no other conceptual framework suggests that occupational therapists consider the concepts of IMP in combination when trying to facilitate occupational performance in their practice.

In line with expectations, this study found statistically significant correlations among IMP variables. This finding supports previous qualitative research in which the IMP concepts were presumed to have had theoretically-based relationships [7-9]. There are several possible explanations to be considered for this result. First is the Fisher's statement that 'purpose can be derived from the meaning one makes of a situation [and] meaning can be derived from one's purpose for engaging in the activity' [24]. Moreover, perception is believed to be fed with meaning [25]. Third, perception is thought to be the prerequisite for shaping intention and meaning, while it was also reported the outcome of preceding intention and meaning [26].

It should be noted that this study has been concerned primarily with the small sample size, so caution must be applied when generalizing the results. The current study adds to a growing body of literature on the wellconceptualized Neuro-occupation model. Moreover, the finding has significant implications for developing an assessment tool based on IMP concepts that would increase the practicality of the model and utility for clinical 
treatment. Additionally, the importance of considering the combined interactive effect of the variables provides some support for the idea of facilitating occupational performance through manipulating the concepts, however further studies are required to test such a claim.

\section{Conclusion}

Based on this finding, it is advisable that IMP variables be taken into account in combination when occupational therapists provide interventions for their clients.

\section{Acknowledgments}

This research that has been financially supported by Shiraz University of Medical Sciences (SUMS), Iran, represents the findings of the study from an approved research project with the grant number of 94-01-06-9879. The authors would like to thank the financial support of SUMS, and the cooperation of participants.

\section{Conflict of Interest}

The authors declare no conflicts of interest.

\section{References}

[1] Turpin MJ, Iwama MK. Using occupational therapy models in practice: A fieldguide. London: Churchill Livingstone; 2010.

[2] Stamm TA, Cieza A, Machold K, Smolen JS, Stucki G. Exploration of the link between conceptual occupational therapy models and the International Classification of Functioning, Disability and Health. Australian Occupational Therapy Journal. 2006; 53(1):9-17. doi: 10.1111/j.1440-1630.2005.00513.x

[3] Lazzarini I. Neuro-occupation: The nonlinear dynamics of intention, meaning and perception. British Journal of Occupational Therapy. 2004; 67(8):342-52. doi: $10.1177 / 030802260406700803$

[4] Lazzarini I. A nonlinear approach to cognition: A web of ability and disability. In: Katz N, editor. Cognition and Occupation Across the Life Span: Models for Intervention in Occupational Therapy. Bethesda: American Occupational Therapy Association; 2005.

[5] Haltiwanger E, Lazzarini I, Nazeran H. Application of nonlinear dynamics theory to Neuro-occupation: A case study of alcoholism. British Journal of Occupational Therapy. 2007; 70(8):349-57. doi: 10.1177/030802260707000805

[6] Piven E, Derakhshanrad SA. A case study demonstrating reduction of aggressive client behaviors using the Neuro-Occupation model: Addressing professional burnout through nonlinear thinking. Occupational Therapy in Mental Health. 2017; 33(2):179-94. doi: 10.1080/0164212X.2017.1278734
[7] Derakhshanrad SA, Piven E, Hosseini SA, Shahboulaghi FM Nazeran H, Rassafiani M. Exploring the nature of the intention, meaning and perception process of the Neuro-occupation model to understand adaptation to change. Occupational Therapy International. 2016; 23(1):29-38. doi: 10.1002/oti.1402

[8] Derakhshanrad SA, Piven E, Zeynalzadeh Ghoochani B. Comparing the cognitive process of circular causality in two patients with strokes through qualitative analysis. Nonlinear Dynamics, Psychology, and Life Sciences. 2017; 21(4):555-67. PMID: 28923161

[9] Derakhshanrad SA, Piven E, Zeynalzadeh Ghoochani B. Adaption to stroke: A nonlinear thinking approach in occupational therapy. Occupational Therapy in Health Care. 2017; 31(3):255-69. doi: 10.1080/07380577.2017.1335922

[10] Carter RE, Lubinsky J, Domholdt E. Rehabilitation research: Principles and applications. Amesterdam: Elsevier Saunders; 2011.

[11] Derakhshanrad SA, Piven E. Modification of the Persian version of Hermans Achievement Motivation Questionnaire to develop an adapted scale for measuring motivation of poststroke survivors in Iran. Iranian Journal of Neurology. 2016; 15(4):189-94. PMCID: PMC5392191

[12] Richardson G. The metatheory of resilience and resiliency. Journal of Clinical Psychology. 2002; 58(3):307-21. doi: 10.1002/jclp.10020

[13] Price P, Kinghorn J, Patrick R, Cardell B. "Still there is beauty": One man's resilient adaptation to stroke. Scandinavian Journal of Occupational Therapy. 2012; 19(2):111-17. doi: 10.3109/11038128.2010.519402

[14] Connor KM, Davidson JR. Development of a new resilience scale: The Connor-Davidson resilience scale (CD-RISC) Depression and Anxiety. 2003; 18(2):76-82. doi: 10.1002/ da.10113

[15] Derakhshanrad SA, Piven E, Rassafiani M, Hosseini SA, Shahboulaghi FM. Standardization of Connor-Davidson Resilience Scale in Iranian subjects with cerebrovascular accident. Journal of Rehabilitation Sciences and Research. 2014; 1(4):73-7.

[16] Law M, Baptiste S, McColl M, Opzoomer A, Polatajko H, Pollock N. The Canadian occupational performance measure: An outcome measure for occupational therapy. Canadian Journal of Occupational Therapy. 1990; 57(2):82-87. doi $10.1177 / 000841749005700207$

[17] Dalvand H, Dehghan L. [Canadian occupational performance measure (Persian)]. Tehran: Ghalame Elm; 2015.

[18] Atashi N, Aboutalebi S, Heidari M, Hosseini SA. Reliability of the Persian version of Canadian Occupational Performance Measure for Iranian elderly population. Iranian Rehabilitation Journal. 2010; 8(2):26-30.

[19] Pallant J. SPSS survival manual: A step by step guide to data analysis using SPSS for windows. New York: Open University Press; 2007

[20] Crabtree JL. On occupational performance. Occupational Therapy In Health Care. 2003; 17(2):1-18. doi: 10.1080/ J003v17n02_01

[21] Gage M, Polatajko H. Enhancing occupational performance through an understanding of perceived self-efficacy. 
American Journal of Occupational Therapy. 1994; 48(5):45261. doi: 10.5014/ajot.48.5.452

[22] Crabtree JL. No one dresses accidentally: A research synthesis on intentional occupational performance. OTJR: Occupation, Participation and Health. 2010; 30(3):100-10. doi: 10.3928/15394492-20090725-01

[23] Persson D, Erlandsson LK, Eklund M, Iwarsson S. Value dimensions, meaning, and complexity in human occupation-a tentative structure for analysis. Scandinavian Journal of Occupational Therapy. 2001; 8(1):7-18. doi: $10.1080 / 11038120119727$

[24] Fisher AG. Uniting practice and theory in an occupational framework. American Journal of Occupational Therapy. 1998; 52(7):509-521. doi: 10.5014/ajot.52.7.509

[25] Merleau-Ponty M. Phenomenology of perception. New York: Routledge; 2002.

[26] Freeman WJ. Consciousness, intentionality and causality. Journal of Consciousness Studies. 1999; 6(11-12):143-72. 
